\title{
The Effective Temperature and the Absolute Magnitude of the Stars
}

\author{
Angel Fierros Palacios \\ Instituto de Investigaciones Eléctricas, División de Energías Alternas, Mexico \\ Email: afierros@iie.org.mx \\ Received 4 June 2015; accepted 8 January 2016; published 12 January 2016 \\ Copyright (C) 2016 by author and Scientific Research Publishing Inc. \\ This work is licensed under the Creative Commons Attribution International License (CC BY). \\ http://creativecommons.org/licenses/by/4.0/

(c) (i) Open Access

\begin{abstract}
The theoretical framework developed by A.S. Eddington for the study of the inner structure and stability of the stars has been modified by the author and used in this work to show that knowing the effective temperature and the absolute magnitude, the basic parameters of any gaseous star can be calculated. On the other hand, a possible theoretical explanation of the Hertzsprung-Russel Diagram is presented.
\end{abstract}

\section{Keywords}

The Effective Temperature and the Absolute Magnitude of the Stars

\section{Introduction}

The study and understanding of the deep inner parts of the Sun and other stars is a physical situation which seems to be outside the reach of traditional scientific research methods. However, these celestial objects are continuously sending out information to the outer space through the material barriers inside themselves, and this information can be registered in the form of observational data. Therefore, it can be said that the interior of the stars is not disconnected from the rest of the Universe. The gravitational field generated by the stars interacts with other material celestial objects, according to a perfectly determined law. Furthermore, radiant energy coming from the hot interior of the stars reaches the surface after undergoing many deviations and transformations, and begins its journey to outer space. Thus, the dynamic mechanisms inside the stars produce energy processes with results that manifest themselves on the outer part of the star. These results translate into observational data which, along with the general principles governing natural processes such as the conservation of energy and momentum, the laws of thermodynamics, the fundamental properties of radiation and the atom, etc., constitute a basic physical scheme for the construction of a closed and complete theory. 
In posing the problem it is important to propose that the material which makes up the stars is always considered as having a fixed composition, with temperature and mass density values increasing inwards, towards the interior of the star. Additionally, the star configuration is considered to have spherical symmetry, with an open free surface which is the stellar atmosphere. The physical state of that atmosphere depends on certain extraneous influences to which it is subject, such as the gravity force which compresses the material, the flux of radiant heat which flows out from the deeper interior of the star, and the self-generated magnetic field which prevents the star from collapsing or exploding [1].

In order for the star's atmosphere to remain in a steady state, it will have to be self-adjusted so that radiant heat is allowed to go through. Under these circumstances it can be assured that the surface conditions depend on two parameters, namely, the value of the effective temperature $T_{e}$ and that of gravity $g$. When these two control factors vary, the state of the star atmosphere can also vary in two directions, originating that the possible varieties of stellar spectra form a twofold sequence; that is, one gets a two-dimensional array centered on two criteria. At the beginning and for a long time only a one-dimensional order was recognized, e.g. the well known sequence of the spectral types; but the spectroscopic method of determining absolute magnitudes, introduces a classification of spectra transverse to the classification of the spectral types.

Roughly speaking, the criterion of spectral types follows the parameter $T_{e}$, and the absolute magnitude criterion the parameter $g$. The observational criteria divide the two-dimensional distribution of states into one system of meshes, and the parameters $T_{e}$ and $g$ into another system.

The same twofold sequence of possible states appears when the star is considered as a whole. Evidently one sequence is obtained by considering stars of different mass. A transverse sequence is formed by stars of the same mass but different radius or mean density. Thus, a third way of dividing into meshes the two-dimensional distribution of states is obtained when the mass $M$ and the radius $R$ are taken as the parameters.

Consider the connection between the three pairs of parameters, any pair defining a unique state of the star. The connection of the spectral criteria with $g$ and $T_{e}$ is a problem of great importance which apparently has nothing to do with what occurs inside the stars. As regards the connection of $g$ and $T_{e}$ with $M$ and $R$, the connection of $g$ does not deserve further comment. The important question is centered on the way the stellar mass and radius determine the value of $T_{e}$ or, equivalently, the rate of outflow of radiation. This is essentially a problem of the stellar interior and not of the surface conditions. A star does not radiate a certain amount of energy per unit area and per unit time because its photosphere is at a given effective temperature; on the contrary, its photosphere is found at that temperature because that energy flux is passing through it. The intensity of the energy flux coming from inside the star and flowing through its surface depends on two factors: one which helps the flow and another one which hinders it. It is well known that heat flows from regions of higher temperature to regions of lower temperature; thus, thermal flows inside the star must be attributed to the existence of a thermal gradient originated by the gradual increment of temperature from the surface inward. The hindering factor is the obstruction opposed by the stellar material to the transmission of this stream of heat. Since, on the other hand, it is generally acknowledged that the heat generated inside the star is transmitted outward almost entirely by radiation, the obstruction found by the radiant heat flow on its journey to the exterior of the star is called opacity, opacity coefficient, or absorption coefficient of the stellar material.

As will be seen in the following sections, the values of the main parameters of any gaseous star can be obtained by direct calculation with only two observational data, such as the effective temperature and the bolometric or absolute magnitude.

\section{The Stellar Radius, the Effective Temperature, and the Absolute Magnitude}

The relationship existing between the radius of any gaseous star, its effective temperature and its absolute or bolometric magnitude, is found in the magnitude of the self-generated magnetic field at the surface of the star [1]. It can be shown that the absolute value of that magnetic field is as follows [1]:

$$
H=m T_{e}^{2},
$$

with

$$
m=\left(\frac{8 \pi a}{3}\right)^{1 / 2}=2.53 \times 10^{-7} \text { gauss } \cdot \mathrm{K}^{-2}
$$


being a constant.

On the other hand, from the well known relationships [1]-[3]

$$
L=\pi a c R^{2} T_{e}^{4}
$$

and

$$
\log \frac{L}{L_{\odot}}=\frac{M_{b o l \odot}-M_{b o l}}{2.512},
$$

where $L$ is the luminosity, $a$ is the constant of the Stefan law [1] [2], $c$ is the speed of light in vacuum space and the symbol $\odot$ refers to the parameters of the Sun considering it as the unit of measure, and substituting (3) in (4) and taking (1) into account, it can be directly shown that

$$
\log \left(\frac{R H}{R_{\odot} H_{\odot}}\right)=\frac{M_{b o l \odot}-M_{b o l}}{5.024} .
$$

The radius $R$ can be easily calculated with the help of relationships (1), (2) and (5). The luminosity can then be calculated from formula (3) using the value of the effective temperature.

\section{The Effective Temperature and the Stellar Mass}

In order to determine de mass of any gaseous star, it is first necessary to calculate the value of the $\beta$ parameter for such star. This parameter is defined as follows [1] [2] [4]

$$
\begin{aligned}
& p_{r}=(1-\beta) p \\
& p_{g}=\beta p ;
\end{aligned}
$$

where $p_{r}(x, t)$ is the pressure from the radiation, $p_{g}(x, t)$ is the pressure from the hot gases and $p(x, t)$ is the total pressure. Next, lets consider the modified mass-luminosity relationship [1]

$$
L=\frac{4 \pi G c M}{\alpha k_{c}}\left(\frac{1-\beta}{\beta}\right)
$$

where $G$ is the gravitational constant, $M$ is the stellar mass, $k_{c}$ is the opacity coefficient calculated at the center of the star and $\alpha=2.5$ is a constant indicating an intense and uniform concentration of energy generating sources at the center of the star [1] [2]. It is important to indicate that for numerical calculations $k_{c}=k$ is usually assumed [1] [2]. The relationship (7) can be written as follows

$$
\begin{aligned}
L & =\frac{4 \pi G c(1-\beta) M}{\alpha k_{1} \beta} \cdot \frac{T^{3}}{\rho} \cdot T^{1 / 2} \\
& =\frac{4 \pi G c}{\alpha k_{1}} \cdot \frac{3 R}{a \mu}\left[\frac{G}{4 R} \cdot \frac{R^{\prime}}{M^{\prime}} \frac{\mu}{R}\right]^{1 / 2} \cdot M^{3 / 2} \cdot \frac{(1-\beta)^{2}}{\beta^{2}},
\end{aligned}
$$

where the following relationship was used [1] [2]

$$
k=k_{1} \rho T^{-7 / 2},
$$

which is the commonly accepted law for radiation absorption [1] [2]. Furthermore, the following relationship can be used to calculate the temperature at the center of the star [1] [2]

$$
\frac{T^{3}}{\rho}=\frac{3 R(1-\beta)}{a \mu \beta} ;
$$

where $R$ is the gas constant and $\mu$ is the average molecular weight whose numerical value is always taken equal to 2.11 [1] [2]; and 


$$
T=\frac{\mu}{R(n+1)} \cdot \frac{G M}{M^{\prime}} \cdot \frac{R^{\prime}}{R} .
$$

This last alternative relationship to calculate the temperature at the center of the star is obtained from the theory of the polytropic gas sphere [1] [2] [5]. The following numerical results $M^{\prime}=2.015$ and $R^{\prime}=6.901$ are also taken from there for those parameters.

It can be shown that for gaseous stars $n=3$ [1] [2] [6]. Now, from the following equation [2] [6]

$$
1-\beta=C M^{2} \mu^{4} \beta^{4}
$$

with

$$
C=\frac{\pi G^{3} a}{48 R^{4} M^{\prime 2}},
$$

being a constant, the mass $M$ can be eliminated. Combining both equations it can easily be obtained that

$$
M^{3 / 2}=\left[\frac{48 R^{4} M^{\prime 2}}{\pi G^{3} a}\right]^{3 / 4} \cdot \frac{(1-\beta)^{3 / 4}}{\mu^{3} \beta^{3}} .
$$

In that case, one gets in (8) that

$$
L=\frac{\pi a c G}{\alpha k_{1}} \cdot \frac{3 R}{a} \cdot\left[\frac{G}{4 R} \cdot \frac{R^{\prime}}{M^{\prime}}\right]^{1 / 2} \cdot\left[\frac{48 R^{4} M^{\prime 2}}{\pi G^{3} a}\right]^{3 / 4} \cdot \frac{(1-\beta)^{11 / 4}}{\mu^{7 / 2} R^{1 / 2} \beta^{5}} .
$$

With the numerical values of the constants (see Appendix) substituted in (14) the following result can be obtained, which is valid for any gaseous star [1] [6]

$$
L=\frac{1.443 \times 10^{71}(1-\beta)^{11 / 4}}{\alpha k_{1} R^{1 / 2} \mu^{7 / 2} \beta^{5}} .
$$

According to the previous affirmation, any two stars can be compared if one of them is considered as the appropriate unit of measure. In this case it is common practice to use the brightest component of the Capella binary system [1] [2]; for which the numerical values of its basic parameters are known. Consequently, the following formula can be obtained from equation (15)

$$
\frac{L_{*}}{L}=\left(\frac{k_{1}}{k_{1^{*}}}\right) \cdot\left(\frac{R}{R_{*}}\right)^{1 / 2} \cdot\left[\frac{1-\beta_{*}}{1-\beta}\right]^{1 / 4} \cdot\left(\frac{\beta}{\beta_{*}}\right)^{5}
$$

where the asterisk refers to the particular star which is being compared to Capella. With the help from the data for this last star (see Appendix) one gets

$$
\left[\frac{1-\beta_{*}}{1-\beta}\right]^{11 / 4} \cdot\left(\frac{\beta}{\beta_{*}}\right)^{5}=6.0981 \frac{\left(1-\beta_{*}\right)^{11 / 4}}{\beta_{*}^{5}}
$$

such that in (16) one gets the following

$$
L_{*} R_{*}^{1 / 2}=\left(\frac{k_{1}}{k_{1^{*}}}\right) \cdot 2.86 \times 10^{42} \cdot \frac{\left(1-\beta_{*}\right)^{11 / 4}}{\beta_{*}^{5}} .
$$

Since the effective temperature is a conventional measure used to specify the flow of radiant heat emitted per unit area by any star [1] [2], it is proposed here as a working hypothesis that, for gaseous stars, constants $k_{1}$ and $k_{1 *}$ appearing in equation (18), keep the same proportion among themselves as that of their respective effective temperatures. In such case, it will be considered that the ratio $k_{1} / k_{1^{*}}$ and $T_{e} / T_{e^{*}}$ are numerically equal, such that 
from this point of view, it is always true that

$$
\frac{k_{1}}{k_{1^{*}}}=\frac{T_{e}}{T_{e^{*}}} ;
$$

where, evidently, $k_{1}$ is the constant in equation (9) for the case of Capella. Consequently, from relationship (18) the following numerical equation for the calculation of parameter $\beta_{*}$ for any gaseous star is obtained

$$
\left(1-\beta_{*}\right)^{11 / 4}=\mathcal{A}_{*} \beta_{*}^{5} ;
$$

where

$$
\mathcal{A}_{*}=\left(L_{*} T_{e^{*}} R_{*}^{1 / 2}\right) \times 0.67 \times 10^{-46},
$$

is a constant for each particular star under study.

Once the parameter $\beta_{*}$ has been obtained, the following fourth degree equation is used to determine the mass of the star [1] [2]; considering that $\mu=2.11$

$$
1-\beta_{*}=3.09 \times 10^{-3}\left(M_{*} / \odot\right)^{2} \mu^{4} \beta_{*}^{4} .
$$

In this way, one gets all the necessary results to calculate the volume, the average and central mass density, as well as the opacity coefficient and the temperature at the center of the star. The magnitude of the self-generated magnetic field can also be estimated at any point inside the star.

\section{V Puppis}

As an illustrative example, lets consider the case of the brightest component of the binary system V Puppis. According to observational data, that star is an eclipsing variable of spectral class $B_{1}$. Its effective temperature is equal to $1.9 \times 10^{4} \mathrm{~K}$; while its bolometric magnitude is -4.75 and its visual magnitude -3.12 [2]. With the help of the formulas from paragraph 1 , it is easy to calculate that

$$
\begin{aligned}
& H_{*_{S}}=91.3 \text { gauss } \\
& R_{*}=5.33 \times 10^{11} \mathrm{~cm} . \\
& L_{*}=2.67 \times 10^{37} \mathrm{ergs} \cdot \mathrm{seg}^{-1} ;
\end{aligned}
$$

such that from (20) the following numerical equation is obtained

$$
25 \beta_{*}^{5}=\left(1-\beta_{*}\right)^{11 / 4} .
$$

Using Newton's method [7] for the solution of numerical equations, the following results are obtained

$$
\begin{aligned}
& \beta_{*}=0.408 \\
& 1-\beta_{*}=0.592 .
\end{aligned} .
$$

Substituting these data in (22) one gets that

$$
M_{*}=18.7 \odot=3.71 \times 10^{34} \text { gr. }
$$

Since the volume of the star is

$$
V_{*}=\frac{4}{3} \pi R_{*}^{3}=6.34 \times 10^{35} \mathrm{~cm}^{3} .
$$

Its average density is

$$
\rho_{m *}=0.0585 \mathrm{gr} \cdot \mathrm{cm}^{-3} .
$$

It can be seen from the theory of the polytropic gas sphere [1] [2] that the central density is given by the following relationship 


$$
\rho_{c^{*}}=54.36 \rho_{m^{*}}=3.18 \mathrm{gr} \cdot \mathrm{cm}^{-3} .
$$

Substituting results (25) and (28) in to equation (10) the following value for the central temperature is obtained

$$
T_{c^{*}}=4.12 \times 10^{7} \mathrm{~K} .
$$

On the other hand, and given that $k_{1}=1.26 \times 10^{27}[1]$, from the relationship among the effective temperatures and from (19), it is easy to prove that

$$
k_{1 *}=4.6 \times 10^{27} .
$$

Finally, with the help of results (28), (29) and (30), and from relationship (9), the following value for the opacity coefficient is obtained

$$
k_{c^{*}}=32.6 \mathrm{~cm}^{2} \cdot \mathrm{gr}^{-1} \text {. }
$$

\section{The Self-Generated Magnetic Field}

The magnitude of the self-generated magnetic field in V Puppis can be estimated at any region inside the star with the help of the general relationship [1]

$$
H=\left[\frac{2 \pi \rho \phi(1-\beta)}{\beta}\right]^{1 / 2}
$$

plus the numerical data for $M^{\prime}$ and $R^{\prime}$ obtained from the theory of the polytropic gas sphere [1] [2]. Thus, the magnitude of the gravitational potential at the center of the star can be calculated from the following result [2]

$$
\phi_{c}=\frac{G M}{M^{\prime}} \cdot \frac{R^{\prime}}{R},
$$

such that

$$
\phi_{c^{*}}=\frac{6.66 \times 10^{-8} \times 3.71 \times 10^{34} \times 6.901}{2.015 \times 5.33 \times 10^{11}}=1.59 \times 10^{16} .
$$

In this case,

$$
H_{c^{*}}=\left(\frac{2 \times 3.14 \times 3.18 \times 1.59 \times 10^{16} \times 0.592}{0.408}\right)^{1 / 2}=6.79 \times 10^{8} \text { gauss. }
$$

According to the results from the theory of the polytropic gas sphere [2], it is seen that point $z=3.5$ indicates a position placed a bit beyond half the distance from the center to the surface of the star. It is easy to verify that with this choice over $90 \%$ of its mass is being accounted for [2]. In this position the gravitational potential has the following value

$$
\phi_{*}=\frac{6.66 \times 10^{-8} \times 3.71 \times 10^{34} \times 3.5}{1.8203 \times 5.33 \times 10^{11}}=8.9 \times 10^{15} .
$$

While also at the same place the mass density is

$$
\rho_{*}=0.02109 \rho_{c^{*}}=0.0671 \mathrm{gr} \cdot \mathrm{cm}^{-3} .
$$

With these results, one gets that

$$
\rho_{*} \phi_{*}=5.97 \times 10^{14} \text {. }
$$

Consequently, the self-generated magnetic field has the following magnitude at that position 


$$
H_{*}=\left(\frac{2 \times 3.14 \times 5.97 \times 10^{14} \times 0.592}{0.408}\right)^{1 / 2}=7.37 \times 10^{7} \text { gauss. }
$$

If $H_{*}$ is compared to $H_{c^{*}}$, then it is easy to verify the fact that $H_{*}=0.11 H_{c^{*}}$

is also valid for $V$ Puppis [1]. The following graph showing the general behavior of the self-generated magnetic field of this star was obtained with the previous values and the one derived from Equation (1) (Figure 1).

To end this paragraph, the distance at which V Puppis is found can be estimated using the visual and bolometric magnitudes from the following expression [8]

$$
m_{v}-M_{b o l}=5.024 \log \frac{r}{10} ;
$$

where $r$ is the distance in parsecs. From the direct calculation it is obtained that this system is found at 427 parsecs from Earth; such distance being equivalent to 1391 light-years.

\section{The Effective Temperature and the Constant $k_{1}$}

By direct calculation from the law of radiation absorption (9) and from relationship (10), it can be shown that

$$
k_{1}=\text { constant } \cdot\left(\frac{1-\beta}{\beta}\right) \cdot k_{c} T_{c}^{1 / 2}
$$

where $k_{c}$ and $T_{c}$ are, respectively, the opacity coefficient and the temperature calculated at the center of the star, and

$$
\text { constant }=\frac{3 R}{a \mu} .
$$

According to definitions (6), the ratio $(1-\beta) / \beta$ is equal to the ratio between the pressure from the radiation and that from the hot gases, $p_{r} / p_{g}$. The numerical value of this ratio for a particular star, is used to get an idea on its degree of stability [2]. On the other hand, the product $k_{c} T_{c}^{1 / 2}$ is a combination between the factor that helps the thermal flow on its way towards the stellar atmosphere, and the factor opposing such flow. Therefore, such combination not only determines the magnitude of the flow of radiant heat emitted through the surface to outer space; but also sets the thermodynamic state for the photosphere to keep its effective temperature at the level corresponding to that energy flow. Hence, it can be stated that for any gaseous star, and for a given value of the average molecular weight, there is a very close relationship between the thermodynamic state at which its photosphere is kept, and the dynamic and energy processes generated in its interior.

Since result (37) remains valid for any gaseous star, the following comparison can be made

$$
\frac{k_{1}}{k_{1 *}}=\left(\frac{k_{c}}{k_{c *}}\right) \cdot\left(\frac{T_{c}}{T_{c *}}\right)^{1 / 2} \cdot \frac{(1-\beta) \beta_{*}}{\left(1-\beta_{*}\right) \beta} .
$$

where again, quantities without an asterisk belong to the brightest component of the binary system Capella; while the asterisk is used to indicate parameters from any other star for which its numerical values are known.

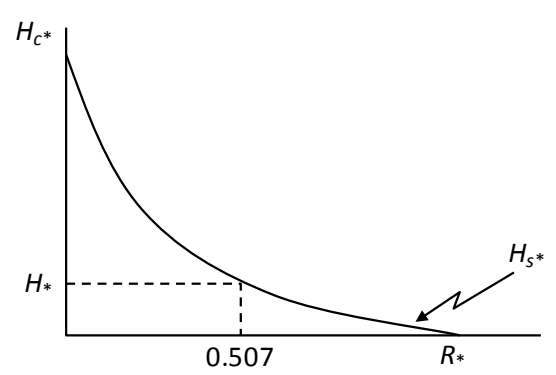

Figure 1. V Puppis' magnetic field. 
For instance, in the case of V Pupp is one has that

$$
\frac{k_{1}}{k_{1 *}}=0.2704 \quad y \quad \frac{T_{e}}{T_{e *}}=0.2737 .
$$

In the case of the Sun, the following results are obtained

$$
\frac{k_{1}}{k_{1 \odot}}=0.9028 \quad y \quad \frac{T_{e}}{T_{e \odot}}=0.9058 .
$$

Finally, given that Capella and $\delta$-Cepheid have the same effective temperature, the following relationship is valid

$$
\frac{k_{1}}{k_{1 \delta}}=\frac{T_{e}}{T_{e \delta}}=1
$$

As it can easily be seen, the matching is complete when the effective temperatures are equal. When they are not, the differences start at the third decimal place. Therefore, it is consideredthattheworkinghypothesisisvalidandveryusefultoclosetheproposedtheory.

\section{Conclusion}

It can be assured, from the solution to the problem previously posed, that the physical state of the stellar surface is determined by the dynamic and energy processes generated inside the star. The magnitude of the thermal radiation flow passing through the surface sets the energy conditions of the stellar atmosphere. Therefore, the effective temperature is the vehicle used by the star to send information to outer space on what is happening in its deeper interior. It is also concluded that the magnitude of the self-generated magnetic field of the star and calculated at its surface, and taking the criterion of the bolometric magnitude as intermediate datum, is the link between the effective temperature and the radius or the stellar mean density. Additionally, it is concluded that the effective temperature is also the important factor to determine the mass and the opacity coefficient of the star, through the parameter $\beta$ which is the basic element relating those two parameters. On the other hand, this is a way of giving a theoretical explanation to the Hertzsprung-Russel Diagram.

\section{References}

[1] Fierros Palacios, A. (2015) The Magnetic Field in the Stability of the Stars. Journal of High Energy Physics, Gravitation and Cosmology, 1, 88-113. http://dx.doi.org/10.4236/jhepgc.2015.12008

[2] Eddington, A.S. (1988) The Internal Constitution of the Stars. Cambridge University Press, New York-New RocheleMelbourne-Sidney. http://dx.doi.org/10.1017/cbo9780511600005

[3] Shwarzchild, M. (1965) Structure and Evolution of the Stars. Dover Publications, Inc., New York.

[4] Chandrasekhar, S. (1958) An Introduction of the Study of Stelar Structure. Dover Publications, Inc., New York

[5] Emden, R. (1907) Gaskugeln: Anwendungen der Mechanischen Wärmetheorie. B.G. Tewbner, Leipzig and Berlín.

[6] Fierros Palacios, A. (2006) The Hamilton-Type Principle in Fluid Dynamics. Fundamentals and Applications to Magnetohydrodynamics, Thermodynamics, and Astrophysics. Springer-Verlag, Wien.

[7] Leonard, E.D. (1964) New First Course in the Theory of Equations. John Wiley \& Sons, Inc., New York-London

[8] Adell, G. (1964, 1969) Exploration of the Universe. Hult, Rinehart and Winston, New York, Chicago, San Francisco, Atlanta, Dallas, Montreal, Toronto, London, Sidney. 


\section{Appendix}

Physical and astronomical constants

$$
\begin{aligned}
& G=6.66 \times 10^{-8} \mathrm{ergs} \cdot \mathrm{cm} \cdot \mathrm{gr}^{-2} \\
& \mathbb{R}=8.26 \times 10^{7} \mathrm{ergs} \cdot \mathrm{mol} \cdot \mathrm{gr}^{-1} \cdot \mathrm{K}^{-1} \\
& a=7.64 \times 10^{-15} \mathrm{ergs} \cdot \mathrm{cm}^{-3} \cdot \mathrm{K}^{-4} \\
& c=3 \times 10^{10} \mathrm{~cm} \cdot \mathrm{sec}^{-1}
\end{aligned}
$$

Relevant data for Capella are the following

$$
\begin{aligned}
& T_{e}=5.2 \times 10^{3} \mathrm{~K} \\
& R=9.55 \times 10^{11} \mathrm{~cm} \\
& L=4.8 \times 10^{35} \mathrm{ergs} \cdot \mathrm{sec}^{-1} \\
& \beta=0.717 \\
& 1-\beta=0.283 \\
& k_{c}=69 \mathrm{~cm}^{2} \cdot \mathrm{gr}^{-1} \\
& T_{c}=9.08 \times 10^{6} \mathrm{~K}
\end{aligned}
$$

